\title{
La contribution française à l'alimentation en eau potable de Sydney
}

\author{
French contribution to the supply of drinking water to Sydney
}

\author{
par P. Mazounie \\ Suez Lyonnaise des Eaux \\ J.-M. Ponte \\ Suez Lyonnaise des Eaux \\ P. Alla \\ Australian Water Services
}

With a capacity of 3 million $\mathrm{m}^{3}$ per day of drinking water (in the future 4,2 million $\mathrm{m}^{3}$ ), the Prospect plant is the largest drinking water treatment works in the world to have been built in one stage. This unit has been developed and is operated by Australian Water Services (Lyonnaise des Eaux) under a BOOT contract with Sydney Water. It was commissioned in September 1996, 30 months after commencing the work.

This paper describes the technical developments in hydraulics, chemical mixing, high rate filtration, automation and highlights the results obtained after a year of operation.

\section{INTRODUCTION}

A la suite d'un appel d'offres international lancé par Sydney Water en 1992 dans le cadre d'un programme global d'amélioration de la qualité de l'eau distribuée sur l'agglomération de Sydney, le contrat de Prospect a été attribué à Australian Water Services, un groupement mené par la Lyonnaise des Eaux en association avec ses partenaires australiens, Lend Lease Corporation et P\&O Australia. Il s'agit d'un contrat de type BOOT (Build, Own, Operate, Transfer) comprenant la conception, le financement, la construction et l'exploitation pendant une période de 25 ans d'une usine de traitement d'une capacité de production journalière de 3 millions de $\mathrm{m}^{3}$ pouvant être augmentée dans le futur à 4,2 millions de $\mathrm{m}^{3}$. Le coût d'investissement de l'ordre de $\mathbf{2 0 0}$ millions de dollars australiens ( 800 millions de francs français) et les coûts d'exploitation sont couverts par le tarif payé par Sydney Water sur le volume d'eau traitée, répondant aux spécifications du contrat, livré en gros au réseau et distribué par Sydney Water au consommateur final.

Mise en service en septembre 1996, après une période de construction de 30 mois seulement et exploitée depuis par Australian Water Services, I'usine de Prospect alimente désormais en eau potable $85 \%$ de l'agglomération de Sydney, soit environ 3 millions d'habitants.

Les principaux enjeux techniques de cette réalisation, qui est aujourd'hui la plus grande usine de production d'eau potable au monde réalisée en une seule phase, étaient lessuivants :

- la conception hydraulique d'ensemble, en raison de l'importance du débit à traiter $\left(50 \mathrm{~m}^{3} / \mathrm{s}\right.$ en phase finale $)$ et d'une hauteur géométrique disponible limitée par des infrastructures existantes :

- le mélange des réactifs avec l'eau brute à traiter, qui conditionne le rendement de la filtration et la qualité finale de l'eau traitée ;

- la filtration proprement dite, compte tenu de sa vitesse nominale élevée ( 23 à $25 \mathrm{~m} / \mathrm{h}$ ) ;

- l'automatisation de l'ensemble des procédés de traitement pour permettre un fonctionnement entièrement automatique de l'usine.

\section{II — LA CONCEPTION GÉNÉRALE DU TRAITEMENT}

La ressource principale d'alimentation en eau brute est le barrage réservoir de Warragamba (lac Burragorong), qui peut délivrer gravitairement un débit allant jusqu'à 2,7 millions de $\mathrm{m}^{3}$ par jour. Les sources d'alimentation d'appoint sont les barrages réservoirs de Nepean au sud de Sydney et le réservoir de Prospect, au bord duquel a été construite l'usine de traitement. 
Ces trois ressources sont particulièrement protégées grâce à une politique de gestion mise en place de longue date par Sydney Water et l'eau brute, qui en résulte, est en général de bonne qualité. Elle peut cependant se dégrader à la suite d'orages violents et prolongés sur les bassins versants. L'eau brute, faiblement minéralisée, se caractérise essentiellement par une turbidité de 0,5 à $25 \mathrm{NTU}$, une couleur d'origine naturelle de 5 à $50^{\circ}$ Hazen, la présence de fer et épisodiquement de manganèse.

La chaîne de traitement est basée sur une filtration directe à forte vitesse sans décantation préalable, qui a été mise au point et optimisée à la suite de campagnes d'essais pilotes approfondies sur site. Elle comprend les étapes successives suivantes :

- une première correction de $\mathrm{pH}$ à la chaux et une oxydation du manganèse soluble par du permanganate de potassium en cas de présence dans l'eau brute ;

- une seconde correction de $\mathrm{pH}$ à l'acide sulfurique, dont la valeur est ajustée en fonction de l'optimum requis pour la coagulation ;

- une préchloration, utilisée uniquement dans des conditions d'eau brute particulièrement difficiles ;

- une coagulation primaire au chlorure ferrique ;

- une coagulation secondaire par dosage d'un polymère cationique avec une nouvelle correction simultanée du $\mathrm{pH}$ par addition d'eau de chaux ;

- un dosage de polymère anionique en tant qu'adjuvant de filtration ;

- la filtration à grande vitesse proprement dite, sur des filtres Degrémont Aquazur $\mathrm{V}$ à sable homogène monocouche ;

- une correction finale du $\mathrm{pH}$ de l'eau filtrée à l'eau de chaux ;

- une désinfection finale au chlore ;

- une fluoruration par dosage de fluosilicate de sodium.

L'eau traitée est stockée dans deux réservoirs d'une capacité totale de $150000 \mathrm{~m}^{3}$, construits à base de matériaux plastiques au lieu de structures traditionnelles en béton, avant de rejoindre le réseau de distribution à l'entrée duquel est appliqué un traitement de chloramination.

Les eaux de lavage des filtres son dirigées vers des épaississeurs gravitaires et les boues issues de cet épaississement sont déshydratées par centrifugation.

\section{III — LES PRINCIPALES INNOVATIONS TECHNIQUES}

\section{- 3.1 La conception hydraulique de l'usine}

La conception hydraulique de l'usine de Prospect, orientée par les infrastructures existantes qui limitaient la hauteur géométrique disponible, répond aux objectifs suivants fixés dès l'origine du projet :

- minimiser, pour des raisons évidentes de compétitivité, la taille des différents ouvrages et en particulier la structure de prise d'eau construite sur un canal existant, les ouvrages de mélange de réactifs, et les filtres proprement dits ;

- accroître la fiabilité de l'usine en mettant en œuvre autant que possible des systèmes de contrôle hydrauliques passifs ; - optimiser l'efficacité du mélange à l'eau brute des différents réactifs ;

- assurer une équirépartition aussi précise que possible du débit entre les différents ouvrages et les filtres en particulier ;
- minimiser les différences de temps de contact entre l'injection de l'adjuvant de filtration et l'arrivée sur les différents filtres ;

- réduire à leur strict minimum les pertes de charges à tous les stades du traitement.

Les solutions mises en place pour atteindre ces objectifs ont résulté d'études sur modèles hydrauliques. Elles comprennent en particulier les aménagements suivants :

- des grilles d'homogénéisation des profils de vitesses dans la structure d'eau brute ;

- des déflecteurs à l'entrée des canaux de contact pour assurer une exacte division du débit entre les quatre modules de filtres et une uniformité des vitesses dans les zones d'injection des réactifs :

- une conception optimisée des chambres de mélange de l'adjuvant de filtration pour obtenir un écoulement uniforme sous les turbines, tout en limitant la perte de charge à son strict minimum ;

- des canaux de section décroissante et vitesse d'écoulement constante pour minimiser les écarts de temps entre l'injection de l'adjuvant de filtration et l'entrée de l'eau sur les différents filtres.

\section{- 3.2 le mélange des réactifs}

Une dispersion rapide et un mélange efficace des réactifs de coagulation et de floculation sont essentiels dans tout traitement de clarification. Ils sont encore plus critiques dans le cas d'une filtration directe pour laquelle le mélange des réactifs et les temps de contact entre les injections constituent le seul moyen de maîtriser la formation et la taille des flocs pour optimiser les performances de la filtration.

Chacun des quatre modules de filtration de l'usine est alimenté par un canal de contact de $4,3 \mathrm{~m}$ de large et $6,5 \mathrm{~m}$ de profondeur d'eau, dimensionné pour un débit ultime de $12,3 \mathrm{~m}^{3} / \mathrm{s}$, dans lequel se font les injections du chlorure ferrique et des polymères cationique et anionique.

Des mélangeurs hydrauliques ont été retenus pour l'injection du coagulant minéral et organique. Ce type de mélangeur n'ayant jamais été réalisé à une telle échelle, ils ont fait l'objet d'une étude de développement spécifique, incluant la réalisation d'un prototype testé à l'échelle industrielle sur des ouvrages de traitement existants. La conception finale comprend 15 diffuseurs quadrillant la section de chaque canal : les jets d'eau de dispersion sont projetés à une vitesse de $12 \mathrm{~m} / \mathrm{s}$ contre des cibles profilées au centre desquelles est injecté le réactif dilué.

Ce type de mélangeur présente les avantages suivants par rapport à des mélangeurs mécaniques classiques :

- une dispersion complète du réactif dans l'eau à traiter obtenue en 1 seconde au débit nominal ;

- une faible perte de charge inférieure à $50 \mathrm{~mm}$ d'eau ;

- un ajustement facile de l'énergie de mélange par réglage du débit d'eau motrice ;

- la possibilité de modifier relativement simplement la position relative des grilles d'injection dans chaque canal pour ajuster les temps de contact entre l'introduction des deux coagulants ;

- une consommation énergétique comparable ;

- une fiabilité accrue, les seuls éléments en rotation étant les pompes d'eau motrice pour lesquelles des secours sont prévus.

On a fait appel à des mélangeurs rapides classiques (mis en œuvre dans des chambres dont l'hydraulique a été optimi- 
sée) pour l'injection de l'adjuvant de filtration, le mélange hydraulique provoquant une rupture partielle des chaînes du polymère sur ce type d'application.

\subsection{La filtration}

L'usine de Prospect comporte 24 filtres répartis en 4 modules de 6 filtres chacun, offrant une surface totale de filtration de $5710 \mathrm{~m}^{2}$ pour un débit nominal de 3 millions de $\mathrm{m}^{3}$ par jour. L'extension future à 4,2 millions de $\mathrm{m}^{3}$ par jour sera obtenue par la construction de 2 filtres supplémentaires sur chaque module, ce qui portera leur nombre total à 32 .

L'adoption d'une vitesse de filtration très élevée qui varie de 23 à $25 \mathrm{~m} / \mathrm{h}$ au débit nominal, soit environ le double de celle couramment appliquée sur des installations de filtration conventionnelles, a permis une réduction très significative de la surface de filtration et par conséquent du coût de construction.

Les filtres du type Aquazur $\mathrm{V}$ à double cellule, conçus par Degrémont, ont une surface de filtration unitaire de $237.9 \mathrm{~m}^{2}$, ce qui les situe parmi les plus grands filtres jamais réalisés. Un sable homogène avec une forte hauteur de couche $(2,1 \mathrm{~m})$ a été choisi de préférence à un matériau filtrant bicouche pour des raisons de coût mais aussi d'économie sur les volumes d'eau de lavage.

La conception du filtre Aquazur $\mathrm{V}$ a été adaptée pour prendre en compte les enseignements tirés des essais pilotes, ce qui a conduit à :

- la mise en place dans le filtre lui-même de deux canaux latéraux équipés d’orifices calibrés à deux niveaux : en phase de filtration, les orifices supérieurs assurent une répartition longitudinale du débit d'alimentation sur la totalité du filtre, uniformisant le temps de contact dans la tranche d'eau de $2 \mathrm{~m}$ au-dessus du matériau filtrant et permettant une amélioration de la performance du filtre : en phase de lavage. seuls les orifices inférieurs sont utilisés pour créer un courant de balayage de surface, qui accélère l'évacuation des matières vers le canal de récupération des eaux de lavage ;

- l'adoption d'un lavage " haute énergie " air et eau, permettant d'une part de désagréger les flocs denses résultant de l'utilisation conjuguée de polymères organiques au stade de la coagulation-floculation et, d'autre part, de chasser l'air résiduel, qui pourrait, dans des conditions d'eau brute chargée, réduire la durée des cycles de filtration.

De façon à limiter le débit instantané de l'eau de rinçage. chaque cellule est lavée indépendamment. La consommation d'eau de lavage est limitée : moins de $6 \mathrm{~m}^{3} / \mathrm{m}^{2}$ de filtre soit moins de $3 \mathrm{~m}^{3} / \mathrm{m}^{3}$ de matériau.

\section{- 3.4 Une usine entièrement automatique}

L'usine de Prospect a été conçue et réalisée pour fonctionner entièrement automatiquement. Cette orientation technique a été prise pendant la conception de l'usine de façon à réduire au maximum les interventions humaines nécessaires au fonctionnement continu de la production. En réalité, cette usine est gérée et maintenue par une équipe de seulement neuf personnes pendant les heures ouvrées et un seul opérateur en dehors de celles-ci.

\subsection{Principes}

Depuis l'eau brute, jusqu'à l'eau traitée délivrée à Sydney Water en sortie d'usine, tout au long de la chaîne de traitement, l'eau est surveillée en permanence. Afin de garantir une sécurité accrue, les principaux paramètres sont mesurés et analysés par deux capteurs installés à chaque point de mesure. Les informations données par ces analyseurs et capteurs sont traitées par une informatique décentralisée sur les différents procédés de traitement constituant l'usine.

Les produits chimiques de traitement sont préparés et dosés, en continu sur site, entièrement automatiquement. La préparation de chaque réactif est réalisée à concentration constante. Chaque réactif est injecté proportionnellement à un point de consigne réglé par l'opérateur, à une mesure de débit au droit du point d'injection et à une mesure du paramètre corrigée en aval du point d'injection du produit chimique. Les boucles de régulation sont pilotées automatiquement par logiciels. Les algorithmes ont été développés pour que les régulations s'adaptent automatiquement aux changements de qualité de l'eau à traiter et à la variation de qualité des produits de traitement.

Les équipements motorisés sont installés en redondance. Les automatismes locaux assurent une permutation automatique en cas de défaillance d'un composant.

Les filtres sont régulés à niveau constant par un automatisme local qui pilote la vanne aval du filtre. Les lavages de filtres sont déclenchés et réalisés automatiquement sur analyse du temps de filtration, de la turbidité mesurée en sortie de chaque filtre ainsi que sur un seuil d'encrassement.

\subsubsection{Architecture informatique}

Le système d'automatisme en place est basé sur l'utilisation d'automates programmable, de logiciels de supervision installés sur PC et d'une base de données. Les automates programmables de marque Télémécanique au nombre de 15 sont répartis sur les différents procédés de l'usine de façon à rendre autonome chaque étape du traitement. Par souci de fiabilité, les automates programmables sont reliés entre eux par des réseaux de communication redondants.

La salle de commande de l'usine comporte principalement deux postes de supervision installés en redondance et complètement indépendants l'un de l'autre. Une base de données (Oracle) est connectée en ligne sur le système informatique temps réel. Cette base de données archive des mesures, des signalisations, des alarmes venant du procédé ainsi que les mesures faites manuellement dans le laboratoire de l'usine. Une application automatique de génération de rapport d'activité et de facture puise les données dans cette base de données. Des PC distribués dans le laboratoire de l'usine et le bureau du chef d'usine permettent la saisie manuelle de données dans la base ainsi que l'accès aux données archivées.

En complément des besoins propres au fonctionnement du procédé, le système d'information de l'usine est interconnecté avec l'infrastructure de gestion technique centralisée du réseau de distribution de Sydney Water.

De façon à garder le contact avec l'opérateur lors de ses tournées à l'intérieur de l'usine pendant la nuit ou les weekends, le système d'information de l'usine transmet automatiquement, sous forme de texte, les alarmes importantes sur un " pager " porté en permanence par l'opérateur.

Afin de garantir une bonne réactivité du personnel de maintenance qui est de garde en dehors des heures ouvrées, un micro-ordinateur portable est mis à la disposition de la personne d'astreinte. Ce micro-ordinateur peut être connecté à distance via le réseau téléphonique sur le système d'information temps réel de l'usine. Il permet ainsi d'être informé du fonctionnement de l'usine et de pouvoir si nécessaire la piloter à distance. 
Tableau 1: Qualité de l'eau produite par l'usine de Prospect en 1997.

\begin{tabular}{|c|c|c|c|c|c|}
\hline & \multicolumn{2}{|c|}{ Critères contractuels } & \multicolumn{3}{|c|}{ Résultats obtenus } \\
\hline & Objectif & Maximum & Minimum & Maximum & Moyenne \\
\hline $\mathrm{pH}$ & $7,7-8,1$ & $7,65-8,15$ & 7,7 & 8,1 & 7,89 \\
\hline Turbidité (NTU) & $<0,3$ & $\leq 0,5$ & 0,03 & 0,15 & 0,06 \\
\hline Couleur $\left({ }^{\circ} \mathrm{H}\right)$ & $<5$ & $\leq 10$ & 2 & 5 & 3,4 \\
\hline Fer $(\mathrm{mg} / \mathrm{l})$ & $<0,2$ & $\leq 0,3$ & $\leq 0,015$ & 0,035 & $\leq 0,016$ \\
\hline Manganèse $(\mathrm{mg} / \mathrm{l})$ & $\leq 0,02$ & $\leq 0,05$ & $\leq 0,005$ & $\leq 0,005$ & $\leq 0,005$ \\
\hline Aluminium total $(\mathrm{mg} / \mathrm{l})$ & $<0,2$ & $\leq 0,2$ & $\leq 0,025$ & $\leq 0,025$ & $\leq 0,025$ \\
\hline Aluminium dissous $(\mathrm{mg} / \mathrm{l})$ & $<0,1$ & $<0,1$ & $\leq 0,025$ & $\leq 0,025$ & $\leq 0,025$ \\
\hline Trihalométhanes $(\mathrm{mg} / \mathrm{l})$ & $\leq 0,1$ & $\leq 0,2$ & 0,011 & 0,03 & 0,02 \\
\hline
\end{tabular}

\section{IV — LES PRINCIPAUX RÉSULTATS D'EXPLOITATION}

\section{- 4.1 Mise en service de l'usine}

L'usine a été mise en service sur le réseau de distribution avec six mois d'avance sur le programme prévu à la satisfaction de Sydney Water et des consommateurs de l'agglomération de Sydney.

La mise en service de l'usine s'est étalée sur une période de quatre mois, dont 30 jours d'essais contractuels de réception avec Sydney Water, au cours de laquelle l'efficacité des solutions techniques retenues au niveau de la conception a été vérifiée :

- la répartition du débit entre les 24 filtres se situe dans une fourchette de $+/-2 \%$, pour un objectif fixé initialement à $+/-3 \%$ au débit nominal ;

- les mélangeurs hydrauliques assurent une dispersion des réactifs de coagulation dans l'eau brute en moins d'une seconde et les consommations de réactifs à l'échelle industrielle sont conformes à celles qui avaient été déterminées à l'échelle pilote ;

- les filtres ont été testés à leur vitesse de filtration nominale sur différentes qualités d'eau brute en particulier pendant les essais de réception avec Sydney Water, assurant une production d'eau filtrée conforme aux prévisions établies à partir des résultats d'essais pilotes et une turbidité d'eau filtrée inférieure à $0,1 \mathrm{NTU}$;

- durant les 30 jours d'essais contractuels, tous les critères de réception de l'usine ont été atteints sans exception, y compris lors d'une pointe de turbidité de 30 NTU sur l'eau brute.

\subsection{La première année d'exploitation}

Le tableau 1 donne les résultats de qualité d'eau obtenus sur l'installation industrielle sur l'année 1997, en regard des principaux critères de qualité exigés par le contrat.

La plupart des paramètres de qualité sur l'eau traitée sont analysés en continu sur l'eau traitée et font l'objet d'un contrôle systématique en laboratoire plusieurs fois par jour.

Les résultats obtenus, qui sont pour la plupart largement inférieurs aux critères de qualité requis par le contrat, prouvent la fiabilité des procédés et des solutions techniques mises en place sur l'usine.

Le système d'assurance qualité mis en place pour l'exploitation de l'usine a par ailleurs été certifié ISO 9002 début 1997.

\section{$\mathrm{V} \square \mathrm{CONCLUSION}$}

La conception de l'usine de Prospect, qui est la plus grande réalisation au monde de ce type construite ces dernières années, a fait l'objet de nombreux développements techniques pour garantir le succès de ce projet.

L'optimisation de l'hydraulique de l'usine, la mise en œuvre de mélangeurs hydrauliques performants, de filtres Aquazur $\mathrm{V}$, optimisés pour la filtration directe à grande vitesse et d'une automatisation poussée ont conduit à une réalisation très compacte et très compétitive sur le plan économique, qui en fait un modèle du genre.

Depuis sa mise en service, l'usine de Prospect délivre en permanence une eau traitée de haute qualité à la satisfaction de Sydney Water et de ses clients. 